\title{
Sudden Dyspnea after Extubation in General Anesthesia: A Case Report
}

Yi-Lu Z*, Shu-Hui T, Zheng-Mei L and Qing-Xiu W

Department of Anesthesiology, East Hospital, Tongji University, Shanghai, China

*Corresponding author: Yi-Lu Z, Department of Anesthesiology, East Hospital, Tongji University, Shanghai, China, E-mail: 18255354282@163.com

Received date: July 05, 2016; Accepted date: Aug 01, 2016; Published date: Aug 05, 2016

Copyright: @ 2016 Yi-Lu Z, et al. This is an open-access article distributed under the terms of the Creative Commons Attribution License, which permits unrestricted use, distribution, and reproduction in any medium, provided the original author and source are credited.

\begin{abstract}
Acute heart failure is a serious complication after general anesthesia, which has a great impact on the prognosis of patients, and even endanger life. We reported a patient who had a heart surgery history, and the cardiac function at stage III, she has to do a radius fracture emergency surgery, while the acute heart failure performance appeared after the extubation. The purpose of this article is to discuss the appropriate choice of anesthesia and anesthesia management in patients with chronic cardiac insufficiency. This will provide us relevant experience in the management of anesthesia for these patients in the future.
\end{abstract}

\section{Introduction}

Heart failure (HF) can be defined as an abnormality of cardiac structure or function leading to failure of the heart to deliver oxygen at a rate commensurate with the requirements of the metabolizing tissues, despite normal filling pressures.

Acute HF (AHF) is a clinical syndrome characterized by acute exacerbation of HF, which can be expressed as acute performance or chronic HF with acute performance [1,2]. Clinical manifestations is suddenly dyspnea, cough frequently with pink foam sputum. Two lungs auscultation can detect moist rales and wheezes. At the beginning of the attack, there was a transient increase in blood pressure, if the disease did not ease, blood pressure continued to decline until shock. The chronic HF patients in the perioperative period have a larger possibility to induce AHF [3]. For the AHF patients, hypoxia and severe respiratory difficulties is a mortal threat, it need to ease as soon as possible. The following is about the emergency treatment of a patient with $\mathrm{AHF}$ in the perioperative period.

\section{Case Report}

A 61 years old female has a history of hypertension for 8 years, coronary heart disease history for more than 10 years and 20 years vitiligo history. She has long history medications, include irbesartan $150 \mathrm{mg \# qd,} \mathrm{isosobide} \mathrm{mononitrate} \mathrm{tablets} 20 \mathrm{mg \# qd}$, aspirin 25 mg\#qd. She was performed CABG in 2004 and deny chronic diseases such as diabetes, respiratory system etc. The patient allergic to penicillin (penicillin skin test positive). Physical examination showed that the degree of opening mouth was at level 2 with teeth missing, while the cervical activity is normal. The body apart form covered with vitiligo ecchymosis, there was no more obvious abnormality. In other examinations, the echocardiography shows: the left ventricular wall motion is reduced, the left atrioventricular increases, a small amount of mitral regurgitation, left ventricular systolic and diastolic function decrease, $\mathrm{EF}$ is $42 \%$. The electrocardiogram shows sinus rhythm with old antero-septal myocardial infarction. The CT scan of wrist joint is distal comminuted fracture of the distal radius. The patients would be treated with open reduction and internal fixation of the left ulnar and radial fractures under general anesthesia. Cardiovascular department consultation opinion points out that the patient were at high risk, include the thrombosis, it is necessary to closely monitor and pay more attention to the heart function during the perioperative time. The patient taking irbesartan to the day of surgery and discontinued aspirin for 3 days. After routinely fasting for $8 \mathrm{~h}$. we gave her the anesthesia induction drugs including midazolam $1 \mathrm{mg}$, propofol 100 $\mathrm{mg}$, sufentanil $0.15 \mathrm{mg}$, rocuronium $40 \mathrm{mg}$, dexamethasone $10 \mathrm{mg}$ and maintained with propofol $200 \mathrm{mg} / \mathrm{h}$, remifentanil $0.5 \mathrm{mg} / \mathrm{h}$ and 0.4 MAC sevoflurane, no additional muscle relaxants. The anesthesia procedure was smoothly and the laryngeal mask were implanted successfully then. The operation lasted for $1.5 \mathrm{~h}$ and intraoperative fluid infusion is about $600 \mathrm{~mL}$. The vital signs were stable and no vasoactive agents were used during the operation.

The patient awaked about $10 \mathrm{~min}$ after the end of surgery. At the time of extubation, her tidal volume recovered to normal and $\mathrm{PetCO}_{2}$ was also in the normal range, the blood pressure was $160 / 100 \mathrm{mmHg}$, $\mathrm{SpO}_{2}$ was $97 \%$, after the smoothly extubation, the patient cough reflex recovery with communication and respond clearly. While she was complained the phlegm are many, so we gave her suction to no sputum. Five minutes later, we sent the patient from the surgery to the PACU, and then the $\mathrm{SpO}_{2}$ appeared to be reduced to $77 \%$, heart rate was $105 \mathrm{bp}$ and blood pressure was 182/106 $\mathrm{mmHg}$. At the time, the patient complained of chest tightness with dyspnea, the double lungs can be heard wheezes and a little crackles. We first considered it is the acute asthma (it can be caused by excessive stimulation of secretions, or due to the temperature changes). So we take measures to treated with sputum suction. Such as: pressure oxygen. Methylprednisolone 40 $\mathrm{mg}$, mucosolvan $30 \mathrm{mg}$ slowly used in intravenous to antiasthmatic, and aminophylline $20 \mathrm{mg}$, salbutamol by nebulization in expansion of trachea. We also gave arterial monitoring to measure the arterial blood pressure and blood gas analysis $\left(\mathrm{PaCO}_{2}\right.$ is $51 \mathrm{mmHg}$ at the respiratory difficulty time.) Esmolol $80 \mathrm{mg}$ were used discontinuously through the change of heart rate. However, there was no obviously change for the patient.

Observation for $15 \mathrm{~min}$, we found the patient's $\mathrm{SpO}_{2}$ can be maintained at $93 \%$ with the oxygen inhalation under pressure, however, when without the oxygen pressure, the $\mathrm{SpO}_{2}$ showed a progressive decline until $80 \%$, the clinical symptoms did not significantly eased. At the same time we were considering the patient had heart disease, her heart function was at level II, the possibility of 
HF was very large, the dyspnea probably due to HF. Than we gave her emergency catheterization and furosemide $20 \mathrm{mg}$ in intravenous to diuretic. Intravenous injection of $0.4 \mathrm{mg}$ cedilanid, pressurized oxygen, the $\mathrm{SpO}_{2}$ slowly rose to $94 \%$. About $20 \mathrm{~min}$ after the treatment of $\mathrm{HF}$, the patient coughed a large pink foam sputum samples, the total of two times about $50 \mathrm{~mL}$. Then the $\mathrm{SpO}_{2}$ remained at $95 \%$, blood pressure was about $160 / 80 \mathrm{~mm} \mathrm{Hg}$, urine volume are $1000 \mathrm{~mL}$, Observation for $1 \mathrm{~h}$, we put her into ICU to strengthen treatment. In the ICU, the patient's cardiac function recovered to the preoperative, vital signs were stable with nitroglycerin, ambroxol, furosemide and other symptomatic treatment, she returned to the general ward 5 days later, and discharged 10 days after surgery.

\section{Discussion}

Our patient has a history of CABG surgery, with high blood pressure and a history of heart disease. Many studies have shown that the heart disease patients are at higher risk for cardiac complications after surgery. These studies found that different preoperative risk factors were useful in predicting postoperative outcome [3-5]. In order to reduce the influence of anesthesia intubation on hemodynamics, we have choose the laryngeal mask airway with mechanical ventilation, strengthen respiratory monitoring, strictly control the volume of fluid infusion during operation and prevent the AHF. Accordingly, the patient was smoothly and no vasoactive drugs were used during the operation time, the vital signs were normal, and no special treatment was done when extubation.

Since the patient has a history of allergy, we considered the acute asthma when she occurred chest tightness with dyspnea and $\mathrm{SpO}_{2}$ drops, while the patient was then diagnosed as cardiac asthma. There are many similarities in the symptoms of cardiac asthma and allergic asthma, meanwhile there are some differences, it is necessary for us to strengthen the differential diagnosis that in order to achieve symptomatic treatment, prevention and treatment of complications timely.

We consider the cause of AHF in this patient is hypoxia. Heart function and oxygen reserve function is poor (X-ray showed: two lungs inflammatory changes, the change after the chest surgery, increased heart shadow). During the transport process after anesthesia, the increased cardiac motion caused by hypoxia, myocardial contractility decreased significantly, the load on the heart increased which resulting in acute cardiac output plunged, the pulmonary circulation pressure suddenly increased, acute pulmonary congestion, pulmonary edema, etc., such a series of clinical syndrome. In addition, the effect of perioperative anesthetic drugs on myocardial injury was also considered.

Patients who prepared to do general anesthesia should be routine fasting. On the one hand, we need to pay attention to the amount of operative fluid supplement, including normal daily basis requirement and anesthesia preoperative fasting after loss of liquid volume, on the other hand, we should prevent AHF occurred because of fluid replacement. Comprehensive evaluation of patients in liquid is the central venous pressure and invasive arterial pressure monitoring [6] and to strengthen the detection of pulmonary artery catheter, S-T segment $[7,8]$. TEE is not necessary for routine use in non-cardiac surgery. In contrast, an acute, persistent, and life-threatening hemodynamic abnormality monitoring can be used to determine the cause of the disease in an emergency or during the perioperative period.
HF can be defined as an abnormality of cardiac structure or function leading to failure of the heart to deliver oxygen at a rate commensurate with the requirements of the metabolizing tissues, despite normal filling pressures (or only at the expense of increased filling pressures). HF including AHF and chronic HF. AHF is a clinical syndrome characterized by acute exacerbation and exacerbation of HF, which can be expressed as acute or chronic HF with acute occur [1]. Clinical manifestations is suddenly severe breathing difficulties, frequently cough with pink foam sputum. Two lungs can be heard wheezes and a little crackles. At the beginning of the attack, there was a transient increase in blood pressure, if the disease did not ease, blood pressure continued to decline until shock.

Acute left HF need to alleviate as soon as possible, the basic treatment including: patients should be with semi-reclining position or sitting position with high flow nasal catheter oxygen, the serious can take CPAP or BiPAP. Morphine 3-5 mg, furosemide $20-40 \mathrm{mg}$ rapidly intravenous injection in two minutes. The use of aminophylline is to relieve bronchial spasm and cedilanid $0.4-0.8 \mathrm{mg}$ to strengthen the cardiac function. Positive and vasoactive drugs can be given. $\beta$ Blockers can reduce perioperative myocardial ischemia and may reduce the risk of myocardial infarction and death in patients with high risk [7].

The use of high doses of opioids, however, is associated with the need for prolonged postoperative mechanical ventilation, and their use may increase length of stay in the intensive care unit (ICU) [9-10]. All inhaled volatile anesthetic agents have cardiovascular effects, including depression of myocardial contractility and afterload reduction. Volatile anesthesia compared with propofol, midazolam and opioid anesthesia can lowering troponin release, improve left ventricular function. Low dose of sevoflurane (0.25-0.5 MAC) and isoflurane has been shown to provide protection in animal models; however, the dose of volatile anesthetics generated myocardial protective in humans is not accurate.

Two kinds of spinal anesthesia techniques (epidural anesthesia and spinal anesthesia) can lead to sympathetic nerve block and reduce the cardiac preload and post load. But the high level block can lead to lower blood pressure and it is not conducive to the prognosis of heart function. The others point out that epidural anesthesia/analgesia did not decrease death or cardiovascular outcomes but modestly improved pulmonary outcomes compared with the general anesthesia group. At the present stage, the monitored anesthesia care (MAC) which composed of local anesthesia and intravenous anesthetics is gradually being taken seriously. Although this technique can eliminate the adverse effects of the some general anesthesia or spinal anesthesia, but the MAC may lead to myocardial ischemia and heart function of abnormal occurrence rate can increased (when local anesthesia is not satisfied).

In this case, the brachial plexus block anesthesia and general anesthesia have their own advantages and weaknesses. Brachial plexus anesthesia by the practicing anesthesiologist can avoid the influence of several general anesthesia drugs on cardiovascular function and reduce the possibility of the occurrence of HF. However, surgical stress itself can be regard as an independent risk factor for HF. If the sudden HF in the brachial plexus block, the first should be given high flow nasal catheter oxygen, pressure oxygen inhalation or mechanical ventilation if necessary. Some general anesthesia drugs may influence the circulatory function, but it also can directly mechanical ventilation in patients and sedative obviously and reduce the cardiac preload by stress. All anesthesia technologies are required to strengthen the monitoring that can detect early and treat properly. This patient is due 
Citation: Yi-Lu Z, Shu-Hui T, Zheng-Mei L, Qing-Xiu W (2016) Sudden Dyspnea after Extubation in General Anesthesia: A Case Report.

Page 3 of 3

to a slight tension before surgery, at the situation that inform her family that general anesthesia and brachial plexus block on the basis of relevant risks, she still require general anesthesia.

All anesthetic techniques and drugs have known cardiac effects, and perioperative planning should take these factors into account. There is no any optimal anesthetic technique for myocardial protection [11-15]. Therefore, anesthesia and intraoperative monitoring should be comprehensive consideration and be chosen. For the non-cardiac surgery for heart disease, preoperative should improve the relevant examination, fully assess, improve preoperative preparation, intraoperative need to strengthen monitoring, prevention and treatment of all complications, postoperative further strengthen monitoring treatment.

\section{References}

1. Gheorghiade M, Pang PS (2009) Acute HF Syndromes. J Am Coll Cardiol 53: 557-573.

2. McMurray JJV, Adamopoulos S, Anker SD, Auricchio A, Bohm M, et al. (2012) ESC Guidelines for the diagnosis and treatment of acute and chronic HF 2012. Eur Heart J 14: 803-869.

3. Goldman L, Caldera DL, Nussbaum SR, Southwick FS, Krogstad D, et al. (1977) Multifactorial index of cardiac risk in non-cardiac surgical procedures. N Engl J Med 297: 845-50.

4. Rao TLK, Jacobs KH, El-Etr AA (1983) Reinfarction following anesthesia in patients with myocardial infarction. Anesthekdogy 59: 499-505.

5. Steen PA, Tinker JH, Tarhan S (1978) Myocardial reinfarction after anesthesia and surgery. JAMA 239: 2566-2570.

6. Fleisher LA, Eagle KA (2001) Lowering cardiac risk in noncardiac surgery. N Engl J Med 345: 1677-1682.

7. Eagle KA, Berger PB, Calkins H, Chaitman BR, Ewy GA, et al. (2002) ACC/AHA guideline update for perioperative cardiovascular evaluation for noncardiac surgery-executive summary: a report of the American College of Cardiology/American Heart Association Task Force on Practice Guidelines (Committee to Update the 1996 Guidelines on Perioperative Cardiovascular Evaluation for Noncardiac Surgery). J Am Coll Cardiol 39: 542-553.
8. Thys DM (1996) Practice guidelines for perioperative transesophageal echocardiography: a report by the American Society of Anesthesiologists and the Society of Cardiovascular Anesthesiologists Task Force on Transesophageal Echocardiography. Anesthesiology 84: 986-1006.

9. Eagle KA, Brundage BH, Chaitman BR (1996) Guidelines for perioperative cardiovascular evaluation for noncardiac surgery. Circulation 93: 1278-1317.

10. Fleisher LA, Beckman JA, Brown KA, Calkins H, Chaikof E, et al. (2007) ACC/AHA 2007 guidelines on perioperative cardiovascular evaluation and care for noncardiac surgery: a report of the American College of Cardiology/American Heart Association Task Force on Practice Guidelines (Writing Committee to Revise the 2002 Guidelines on Perioperative Cardiovascular Evaluation for Noncardiac Surgery) developed in collaboration with the American Society of Echocardiography, American Society of Nuclear Cardiology, Heart Rhythm Society, Society of Cardiovascular Anesthesiologists ...[J]. J Am Coll Cardiol 50: e159-e242.

11. Leung JM, Goehner P, O'Kelly BF, Hollenberg M, Pineda N (1991) Isoflurane anesthesia and myocardial ischemia. comparative risk versus sufentanil anesthesia in patients undergoing coronary artery bypass graft surgery. The SPI (Study of Perioperative Ischemia) Research Group. Anesthesiology 74: 838-847.

12. Baron JF, Bertrand M, Barre E, Godet G, Mundler O (1991) Combined epidural and general anesthesia versus general anesthesia for abdominal aortic surgery. Anesthesiology 75: 611-618.

13. Christopherson R, Beattie C, Frank SM, Norris EJ, Meinert CL, et al. (1993) Perioperative morbidity in patients randomized to epidural or general anesthesia for lower extremity vascular surgery. Perioperative Ischemia Randomized Anesthesia Trial Study Group. Anesthesiology 79: $422-434$.

14. Slogoff S, Keats AS (1989) Randomized trial of primary anesthetic agents on outcome of coronary artery bypass operations. Anesthesiology 70 : $179-188$

15. Tuman KJ, McCarthy RJ, Spiess BD, Ivankovich AD (1990) Epidural anaesthesia and analgesia decreases postoperative hypercoagulability in high-risk vascular patients. Anesth Analg 70: S414. 